\title{
Evaluating clinical outcomes associated with ectopic pregnancies
}

\author{
Palvi Banotra ${ }^{1 *}$, Adil Rashid ${ }^{2}$
}

${ }^{1}$ Department of Health and Medical Education, Jammu, Jammu and Kashmir, India
${ }^{2}$ Department of Statistics, University of Kashmir, Srinagar, Jammu and Kashmir, India

Received: 25 December 2021

Accepted: 07 January 2022

*Correspondence:

Dr. Palvi Banotra,

E-mail: palvibntr@gmail.com

Copyright: (C) the author(s), publisher and licensee Medip Academy. This is an open-access article distributed under the terms of the Creative Commons Attribution Non-Commercial License, which permits unrestricted non-commercial use, distribution, and reproduction in any medium, provided the original work is properly cited.

\begin{abstract}
Background: Ectopic pregnancy refers to the implantation of a fertilized ovum outside the endometrium. There is no doubt that ectopic pregnancy is a common cause of morbidity; however, the associated mortality has declined significantly over the years due to early diagnosis and timely intervention.

Methods: This prospective study was conducted at Lala Ded Hospital, Srinagar from April 2016-March 2017. All the patients with ectopic pregnancies admitted through accident and emergency unit and through gynaecoloy out-patient department were included in the study. The data on several factors was analyzed like; parity, clinical presentation, risk factors, management and findings at laparotomy, to draw a valid inference. All the patients were managed as per hospital protocol and followed till discharge from the hospital.

Results: The present study revealed that the commonest age group affected with ectopic pregnancies is 20-24 years with majority of patients having previous spontaneous abortion as being high risk factor associated with ectopic pregnancies followed by pelvic inflammatory disease. The most dominant clinical presentation among the study subjects was pain and the commonest site of ectopic pregnancy was ampulla region of fallopian tube. Most of the patients were successfully managed with the help of salpingectomy

Conclusions: We suggest that the possibility of ectopic pregnancies should not be ignored in the reproductive age group especially in patients presenting with severe abdominal pain because delayed diagnosis often halts timely intervention which in turn invite serious health consequences.
\end{abstract}

Keywords: Ectopic pregnancy, Salpingectomy, Ampulla region

\section{INTRODUCTION}

Ectopic pregnancy refers to the implantation of a fertilized ovum outside the endometrium..$^{1-3}$ There is no doubt that ectopic pregnancy is a common cause of morbidity; however, the associated mortality has declined significantly over the years due to early diagnosis and timely intervention. Although there is no precise aetiology for ectopic pregnancy, however, a number of causal factors have been identified which include; the integrity of the oviduct, inflammatory insults, intrauterine devices, surgical manipulation, tubal ligations, salpingitis isthmica nodusa, DES exposure, and induced abortions. Sometimes the quality of ovum and hormonal environment is disturbed due to ovulation induction, fertilization in vitro, delayed ovulation, and transperitoneal ovum migration and the associated risk of attaining ectopic pregnancy increases.

The incidence of ectopic pregnancy varies significantly from time to time and country to country; in Europe, the incidence increased from 11.2 to 18.8 per 1,000 pregnancies from 1976 to 1993 and in US, the ectopic pregnancies increased from 17,800 in 1970 to 88,400 in $1989 .^{5,6}$ In UK there were around 11,000 reported cases of ectopic pregnancy per year with an incidence of 11.5 per 1000 pregnancies and the associated mortality rate was 0.4 per thousand ectopic pregnancies). ${ }^{4}$ The ectopic pregnancy 
can be managed surgically, medically or expectantly. Management to individual patients is tailored on the basis of their presentation and on the severity of their condition, suitability of treatment options and more importantly patient preference. Whatever will be the mode of management, the key is the early diagnosis, resuscitation, timely treatment, and follow-up. Early diagnosis of EP is difficult and needs high index of suspicion by treating physician. The present study has been conducted to determine incidence and clinical profile of patients presenting with EP.

\section{METHODS}

The present prospective study on all cases of ectopic pregnancies has been conducted at Lala Ded Hospital, an associated GMC Hospital, Srinagar from April 2016March 2017. All the patients with ectopic pregnancies admitted through accident and emergency unit and through gynaecoloy out-patient department were included in the study. A detailed history, clinical examination and urinary pregnancy testing was performed in all patients suspected of having ectopic pregnancy.

In suspected cases of ruptured ectopic pregnancy, culdocentesis was performed. Patients presenting with adenexal mass and biochemical pregnancy were examined through transvaginal ultrasonography to localize gestational sac, size of ectopic mass, presence of cardiac activity and any evidence of free fluid in pouch of Douglas. The diagnosis of ectopic pregnancy was precisely made on the basis of several factors like; history, clinical examination, urine pregnancy test, culdocentesis and ultrasound examination. The data on several important and relevant factors was analyzed like; parity, clinical presentation, risk factors, management and findings at laparotomy to draw a valid conclusion. All the patients were managed as per hospital protocol and followed till discharge from the hospital.

\section{RESULTS}

The recorded data was compiled and entered in a spreadsheet (Microsoft excel) and then exported to data editor of SPSS version 20.0 (SPSS Inc., Chicago, Illinois, USA).

Statistical software SPSS (version 20.0) and Microsoft Excel were used to carry out the statistical analysis of data. Continuous variables were expressed as mean \pm SD and categorical variables were summarized as percentages.

In the present study, we observed that there were 53 patients included in the study and the average of patients was observed as $(25.7 \pm 2.71)$ years with majority of patients $(54.7 \%)$ falling in the age interval of (20-24) followed by $(26.4 \%)$ falling in (25-29) years. Majority of the patients $(37.7 \%)$ were having $2^{\text {nd }}$ parity followed by $(24.5 \%)$ patients with first parity and $(15.1 \%)$ with $3^{\text {rd }}$ parity. Only 5 patients $(9.4 \%)$ were having a 4 th order parity or beyond; however (13.2\%) were nulliparous. We observe that among studied patients, the most common clinical presentation was abdominal pain (94.3\%) followed by amenorrhea (81.1\%), dizziness (79.2\%), vaginal bleeding $(52.8 \%)$, shock $(20.8 \%)$, shoulder tip pain $(13.2 \%)$ and $(5.7 \%)$ had diarrhoea as clinical presentation. We observed that the most common site of ectopic pregnancy was ampula accounting for (60.4\%) cases followed by isthmus in $(24.5 \%)$ patients.

We observed that the most common risk factor among studied patients was previous spontaneous abortion (73.6\%), pelvic inflammatory disease $(52.8 \%)$, previous tubal surgery $(24.5 \%)$ and previous induced abortion (20.8\%).

Almost $70 \%$ patients were managed with the help salpingectomy, (11.3\%) received medical management, salpingostomy treatment was given to $(11.3 \%)$ and $(3.8 \%)$ patients were managed with salpingo-oopherectomy. Expectant management was exploited on $(3.8 \%)$ patients.

Table 1: Demographic characteristics of study patients.

\begin{tabular}{|llll|}
\hline Variables & & N & $\%$ \\
\hline \multirow{3}{*}{ Age (years) } & $<20$ & 2 & 3.8 \\
\cline { 2 - 4 } & $20-24$ & 29 & 54.7 \\
\cline { 2 - 4 } & $25-29$ & 14 & 26.4 \\
\cline { 2 - 4 } Parity & $30-34$ & 7 & 13.2 \\
\cline { 2 - 4 } & $\geq 35$ & 1 & 1.9 \\
\hline & Nullipara & 7 & 13.2 \\
\cline { 2 - 4 } & Para 1 & 13 & 24.5 \\
\cline { 2 - 4 } & Para 2 & 20 & 37.7 \\
\cline { 2 - 4 } & Para 3 & 8 & 15.1 \\
\cline { 2 - 4 } & $\geq$ Para 4 & 5 & 9.4 \\
\hline
\end{tabular}

Note: Mean \pm SD (range)=25.7 $\pm 2.71(19-38) ; \mathrm{N}=53$.

Table 2: Clinical presentation of study patients.

\begin{tabular}{|lll|}
\hline Presentations & N & $\%$ \\
\hline Abdominal pain & 50 & 94.3 \\
\hline Amenorrhea & 43 & 81.1 \\
\hline Vaginal bleeding & 28 & 52.8 \\
\hline Dizziness & 42 & 79.2 \\
\hline Shock & 11 & 20.8 \\
\hline Shoulder tip pain & 7 & 13.2 \\
\hline Diarrhoea & 3 & 5.7 \\
\hline
\end{tabular}

Table 3: Site of ectopic pregnancy among study patients.

\begin{tabular}{|lll|}
\hline Sites & $\mathbf{N}$ & $\%$ \\
\hline Ampulla & 32 & 60.4 \\
\hline Isthmus & 13 & 24.5 \\
\hline Fimbriae & 4 & 7.5 \\
\hline Tubo-ovarian & 2 & 3.8 \\
\hline Interstitial/cornual & 1 & 1.9 \\
\hline Broad ligament & 1 & 1.9 \\
\hline Total & 53 & 100 \\
\hline
\end{tabular}


Table 4: Risk factors of ectopic pregnancy among study patients.

\begin{tabular}{|c|c|c|}
\hline Risk factors & $\mathbf{N}$ & $\%$ \\
\hline Previous induced abortion & 11 & 20.8 \\
\hline Pelvic inflammatory disease & 28 & 52.8 \\
\hline Previous tubal surgery & 13 & 24.5 \\
\hline Recanalisation & 1 & 1.9 \\
\hline Previous spontaneous abortion & 39 & 73.6 \\
\hline Previous ectopic pregnancy & 2 & 3.8 \\
\hline Intrauterine contraceptive device & 3 & 5.7 \\
\hline
\end{tabular}

Table 5: Distribution according to treatment among study patients.

\begin{tabular}{|lll|}
\hline Treatments & N & $\%$ \\
\hline Expectant management & 2 & 3.8 \\
\hline Medical management & 6 & 11.3 \\
\hline Salpingectomy & 37 & 69.8 \\
\hline Salpingostomy & 6 & 11.3 \\
\hline Salpingo-oopherectomy & 2 & 3.8 \\
\hline Total & 53 & 100 \\
\hline
\end{tabular}

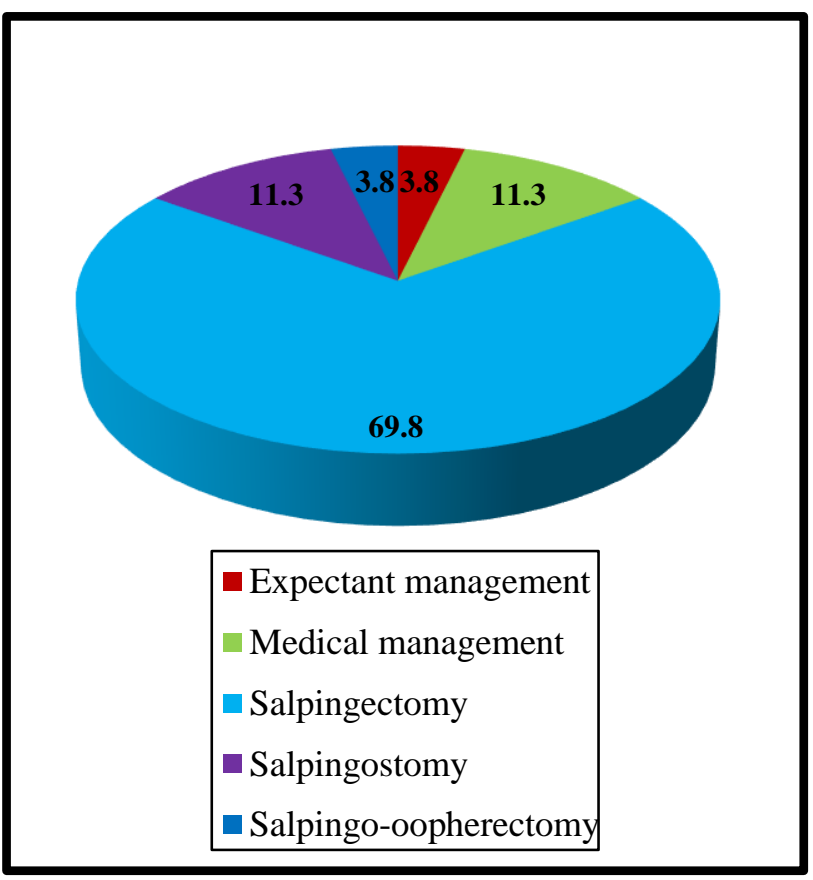

Figure 1: Distribution according to treatment among study patients.

\section{DISCUSSION}

In the present study, we aimed to evaluate the clinical profile of ectopic pregnancies.

We thoroughly analyzed patients data based on demographic, clinical, radiological, surgical, histopathological aspects and follow up data of patients. We randomly included 53 women patients in the study who qualified the inclusion criteria and gave a written consent.
We observed that the average of studied patients was (25.7 \pm 2.71$)$ years with majority of patients $(54.7 \%)$ falling in the age interval of (20-24) followed by $(26.4 \%)$ falling in (25-29) years.

This finding on prevalent age group of ectopic pregnancies patients was not surprising because it always has high risk sexual behavior. In a likewise study by Malik et al, it was reported that the commonest age group affected with ectopic pregnancies is 20-24 years which is quite similar to our result. ${ }^{7}$ However, they reported the average of such patients as $(27 \pm 3)$ years which is contrarily different to their reported prevalent age group of 20-24 years. Contemporarily to the literature, we found that history of previous spontaneous abortion, pelvic inflammatory disease, history of tubal surgery and previous induced abortion are the most common risk factors linked with ectopic pregnancies.

Malik et al have reported the associated risk factors of EP in order of predominance are; history of spontaneous and induced previous abortion, history of pelvic inflammatory disease and tubal surgery which is inconsonance to our observations. ${ }^{7}$ In the present study we found that majority of the patients, accounting for $(37.7 \%)$ were having $2^{\text {nd }}$ parity followed by $(24.5 \%),(15.1 \%)$ patients with first and $3^{\text {rd }}$ parity respectively. Only 5 patients $(9.4 \%)$ were having 4th order parity or beyond; however (13.2\%) were nulliparous. Sindos et al reported that ruptured ectopic pregnancies are associated with parity and previous history of ectopic pregnancy. ${ }^{8}$ With respect to parity, they found that only $(27.1 \%)$ of ruptured cases are nulliparous women (39/144), whereas the respective percentage corresponding to the unruptured cases was $(44.3 \%, 35 / 79)$ and the difference was highly significant with a $(\mathrm{p}$ value $=0.009) .{ }^{8}$

Authors also reported a positive association of borderline statistical significance between previous history of ectopic pregnancy and parity was observed. ${ }^{8}$ In the present study, the most common clinical presentation was abdominal pain $(94.3 \%)$ followed by amenorrhea $(81.1 \%)$, dizziness (79.2\%), vaginal bleeding (52.8\%), shock (20.8\%), shoulder tip pain $(13.2 \%)$ and $(5.7 \%)$ had diarrhea as clinical presentation. The most common site of ectopic pregnancy was ampula accounting for (60.4\%) cases followed by isthmus in $(24.5 \%)$ patients.

Similar kind of observations were made by Malik et al and Stubo et al who reported the commonest site of ectopic pregnancy as ampullary region of fallopian tube. ${ }^{7,9}$ In the present study, most of the patients who had ruptured ectopic pregnancy were managed with emergency laparotomy.

We observed that $(70 \%)$ patients were managed with the help salpingectomy, (11.3\%) were managed medically, few patients $(11.3 \%)$ received salpingostomy treatment and $(3.8 \%)$ patients were managed with salpingooopherectomy. Conservative expectant management was exploited on $(3.8 \%)$ patients. 
Similar to our observation, in a study by Malik et al the commonest surgical procedure performed was also salpingectomy $(65.68 \%)$ and reportedly all of them were having ruptured ectopic pregnancy and likewise to our study they also reported the use medical management in $(10.78 \%)$ patients. Some ectopic pregnancies resolve spontaneously through either regression or tubal abortion, without causing harm to the patient. Expectant management is a conservative strategy consisting of observation and assessment of whether the ectopic pregnancy is continuing to resolve spontaneously and successfully without intervention. ${ }^{10}$ In the present study, the conservative expectant management was exploited on only $(3.8 \%)$ patients which is almost similar to $(4.8 \%)$ reported by Malik et al. ${ }^{7}$

\section{CONCLUSION}

The present study revealed that the commonest age group affected with ectopic pregnancies is 20-24 years with majority of patients having previous spontaneous abortion as being high risk factor associated with ectopic pregnancies followed by pelvic inflammatory disease. The most dominant clinical presentation among the study subjects was pain and the commonest site of ectopic pregnancy was ampulla region of fallopian tube. Most of the patients were successfully managed with the help of salpingectomy. We suggest that the possibility of ectopic pregnancies should not be ignored in the reproductive age group especially in patients presenting with severe abdominal pain because delayed diagnosis often halts timely intervention which in turn invite serious health consequences.

\section{Funding: No funding sources} Conflict of interest: None declared

Ethical approval: The study was approved by the Institutional Ethics Committee

\section{REFERENCES}

1. Walker JJ. Ectopic pregnancy. Clin Obstet Gynecol. 2007;50(1):89-99.

2. Giustina D, Denny M. Ectopic pregnancy. Emerg Med Clin North Am. 2003;21(3):565-84.

3. Varma R, Gupta J. Tubal ectopic pregnancy. BMJ Clin Evid. 2009;2009:1406.

4. Crowhurst JA, Plaat F. Why mothers die--report on confidential enquiries into maternal deaths in the United Kingdom 1994-96. Anaesthesia. 1999;54(3):207-9.

5. Storeide O, Veholmen M, Eide M, Bergsjø P, Sandvei $\mathrm{R}$. The incidence of ectopic pregnancy in Hordaland County, Norway 1976-1993. Acta Obstet Gynecol Scand. 1997;76(4):345-9.

6. Simms I, Rogers PA, Nicoll A. The influence of demographic change and cumulative risk of pelvic inflammatory disease on the incidence of ectopic pregnancy. Epidemiol Infect. 1997;119(1):49-52.

7. Malik R, Jain S, Duhan N, Sirohiwal D. Clinical outcomes of ectopic pregnancy. Int J Reprod Contracept Obstet Gynecol. 2017;6:4277-80.

8. Sindos M, Togia A, Sergentanis TN, Kabagiannis A, Malamas F, Farfaras A, et al. Ruptured ectopic pregnancy: risk factors for a life-threatening condition. Arch Gynecol Obstet. 2009;279(5):621-3.

9. Sotubo O, Aboyeji AP. Ectopic pregnancy in Ilorin, Nigeria: a five-year review. Niger Med Pract. 1994;27:25-7.

10. Condous G, Timmerman D, Goldstein S, Valentin L, Jurkovic D, Bourne T. Pregnancies of unknown location: consensus statement. Ultrasound Obstet Gynecol. 2006;28(2):121-2.

Cite this article as: Banotra $\mathrm{P}$, Rashid A. Evaluating clinical outcomes associated with ectopic pregnancies. Int J Reprod Contracept Obstet Gynecol 2022;11:370-3. 